\title{
BUNCH LENGTH MEASUREMENT IN TIME DOMAIN FOR HLS*
}

\author{
B. Y. Wang", B. G. Sun, J. G. Wang, P. Lu, H. L. Xu, J. H. Wang \\ NSRL, University of Science and Technology of China, Hefei 230029, P. R. China
}

\begin{abstract}
A simple measurement method of beam bunch length in time domain for HLS (Hefei Light Source) has been proposed. The bunch length measurement system is composed of an optical system, a high speed photoreceiver and a wide bandwidth oscilloscope. The photoreceiver which is made by FEMTO has high sensitivity and high bandwidth, which converts the synchrotron radiation light into electronic signal. The oscilloscope which is made by Tektronix is TDS7704B, which has a high bandwidth up to $7 \mathrm{GHz}$ and show the bunch length in time domain. The measurement results of the bunch length and its analysis are given. We compare the results with that measured by the conventional method using a streak camera.
\end{abstract}

\section{INTRODUCTION}

HLS is an $800 \mathrm{MeV}$ electron storage ring with circumference about 66.13 meters in NSRL. The acceleration frequency of HLS is at $204.016 \mathrm{MHz}$. The revolution frequency is $4.534 \mathrm{MHz}$. A variety of filling patterns are possible: 45 bunches (full fill, 100 300mA), single bunch. In full fill pattern, the bunch space is $4.9 \mathrm{~ns}$. In single bunch, the bunch space is $220 \mathrm{~ns}$.

The length of the electron bunches in storage rings is an important parameter to measure, both for machine physicists and for synchrotron light users. Several methods to measure the bunch lengths have been developed, for example, electronic measurement ${ }^{[1]}$, optical measurement ${ }^{[2]}$ and optoelectronic measurement ${ }^{[3]}$. Some studies have been made in HLS, however, the beam signal was picked from the beam position monitor ${ }^{[4-5]}$. We present in this paper another method, in which the time profile of the radiation emitted from the storage ring is measured using photo-receiver and oscilloscope, The bunch length is calculated from the oscilloscope assuming a Gaussian profile for the bunch. It has been shown that the method yields the value in a fair agreement with that measured using a streak camera.

\section{PRINCIPLES}

In storage rings, the temporal profile of the intensity of the synchrotron radiation from a bending magnet is an exact replica of the electron longitudinal distribution within the bunch. This train of very short light pulses can be optically transported up to the acquisition point without appreciable distortion. Then they can be converted into electronic pulses. We can measure the

\footnotetext{
*Work Supported by the Natural Science Foundation of China (10675118) and the CAS Knowledge Innovation Project

\#wby2004@mail.ustc.edu.cn
}

width of the electronic pulse and calculate the width of the synchrotron light pulse.

If the bunch longitudinal distribution is Gaussian, the dependence of the bunch length on the FWHM value of the light pulse is on base of the formula:

$$
\sigma_{\tau}=(F W H M)_{a} / 2.3548
$$

Where $\sigma_{\tau}$ is the bunch length and $(F W H M)_{a}$ is the FWHM value of the synchrotron light pulse.

\section{Bunch Length Calculation}

The pulse width of the electrical signal which converted from the light signal can be measured directly from the oscilloscope. So the synchrotron light passes the optics setup, photo-receiver and the oscilloscope. Considering the rise time caused by the photo-receiver and oscilloscope, the FWHM value of the synchrotron light pulse is expressed as ${ }^{[4]}$

$$
(F W H M)_{a}=\left[(F W H M)_{m}^{2}-\left(2.3548 t_{r} / 2.568\right)^{2}\right]^{1 / 2}
$$

Where $(F W H M)_{a}$ is the FWHM value of the light pulse, $(F W H M)_{m}$ is the FWHM value of the electrical signal converted form the light pulse and $t_{r}$ is the rise time caused by the photo-receiver, the cable and the oscilloscope.

\section{MEASUREMENT SYSTEM DESCRIPTION}

The bunch length measurement system is composed of an optical system, a high speed photo-receiver, a wide bandwidth oscilloscope and a personal computer. The system functional diagram is shown in Fig.1.

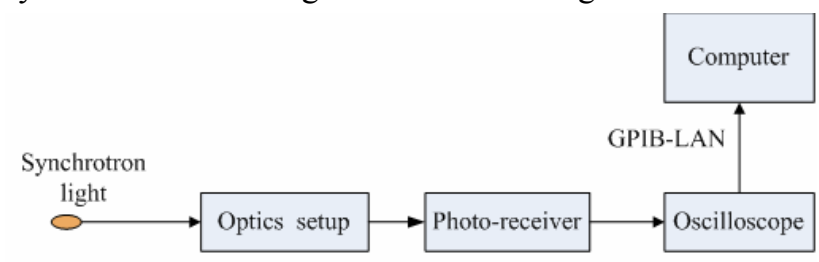

Figure 1: Measurement system setup.

\section{The Optics Setup}

We used the optics setup to extract the synchrotron light from the storage ring vacuum chamber at the bending magnet in the beam diagnostic beam line. The synchrotron light extracting setup is shown in Fig.2.

When the electron beam travel through a bending magnet, it emits the synchrotron light. The synchrotron light is reflected by a copper mirror which is inside the vacuum chamber. The mirror's surface is cooled by water to dissipate the large incident synchrotron radiation power The synchrotron light reflected is transported outside the 
storage ring through a silica window, and go through a set of lens after going through a filter, and then go through a cube beamsplitter and go focused on the active area of the photo-receiver and the streak camera. So, the bunch length can been simultaneously measured by the photoreceiver and the streak camera.

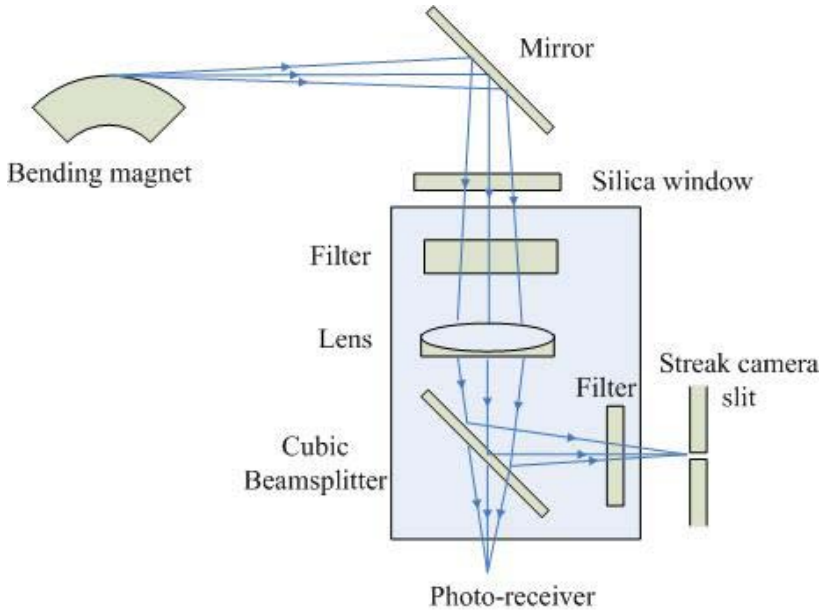

Figure 2: Optics setup.

\section{Photo-receiver}

The high speed photo-receiver is made by FEMTO. Its main parameters ${ }^{[6]}$ are listed in Table 1.

Table 1: Photo-receiver Specification

\begin{tabular}{|l|c|}
\hline Conversion Gain & $4.8 \times 10^{3} \mathrm{~V} / \mathrm{W}(@ 1550 \mathrm{~nm})$ \\
\hline $\begin{array}{l}\text { Frequency } \\
\text { Response }\end{array}$ & $10 \mathrm{KHz} \sim 2 \mathrm{GHz}$ \\
\hline Rise/Fall time & $180 \mathrm{ps}$ \\
\hline Active Area & $\phi 0.2 \mathrm{~mm}$ \\
\hline Spectral Range & $850 \mathrm{~nm} \sim 1700 \mathrm{~nm}$ \\
\hline
\end{tabular}

The synchrotron light which consists of all waveforms with different wave lengths arrives at the active area of the photo-receiver. Then it will be converted into electrical signal. Limited by the spectral range, only part of the light will be converted. The electrical signal converted from the light can be estimated and will be transported to the oscilloscope for measurement. To maximize the signal from the photo-receiver, the photoreceiver is mounted in a three-dimensional micropositioner which has micron resolution.

\section{The Oscilloscope}

The wide bandwidth oscilloscope TDS7704B is made by Tektronix. Its main parameters ${ }^{[7]}$ are listed in Table 2 . It is used to measure the electrical signal from the photoreceiver.

Table 2: Oscilloscope Specification

\begin{tabular}{|l|c|}
\hline Input channels & 4 \\
\hline Rising time $(10 \%-90 \%)$ & $62 \mathrm{ps}$ \\
\hline Bandwidth $(-3 \mathrm{~dB})$ & $7 \mathrm{GHz}$ \\
\hline Input impedance & $50 \Omega \pm 2.5 \%$ \\
\hline Sample rate & $20 \mathrm{GHz} / \mathrm{s}$ \\
\hline
\end{tabular}

06 Instrumentation, Controls, Feedback \& Operational Aspects

\section{DATA PROCESSING AND RESULTS}

\section{Data Processing}

The FWHM value of the electrical signal displayed on the oscilloscope can be obtained through network. The rise time $t_{r}$ which caused by the measurement system is $190.8 \mathrm{ps}$. Then the FWHM value of the synchrotron light pulse can be calculated on basis of formula 2, and the bunch length can be got by formula 1 . Two programs that run on the personal computer were produced to implement the process.

Program one used to control the Tektronix TDS7704B oscilloscope remotely was designed. It used LabVIEW as the programming language and is on basis of the TekVISA supported by the oscilloscope. The TDS7704B oscilloscope can be controlled remotely over a LAN that using VXI-11 protocol and a 10Base-T Ethernet connection together with virtual GPIB software running on the TDS7704B oscilloscope. And TekVISA implements a subset of Version 2.2 of the VISA specification for controlling GPIB and serial (RS-232) instrument interfaces locally or remotely via an Ethernet LAN connection. So we can control the oscilloscope remotely using the program. The interface of the program is shown in Fig.3. We can observe the beam current, the FWHM value of the synchrotron light pulse, the waveform of the electrical signal, the operation status of the machine and the dependence of the bunch length on the beam current. The bunch length measurement system in time domain can monitor the bunch length on-line.

Program two was used to acquire data and write the data into file. The program used the $\mathrm{C} / \mathrm{C}++$ as the programming language. The data in the file can be processed by Origin.

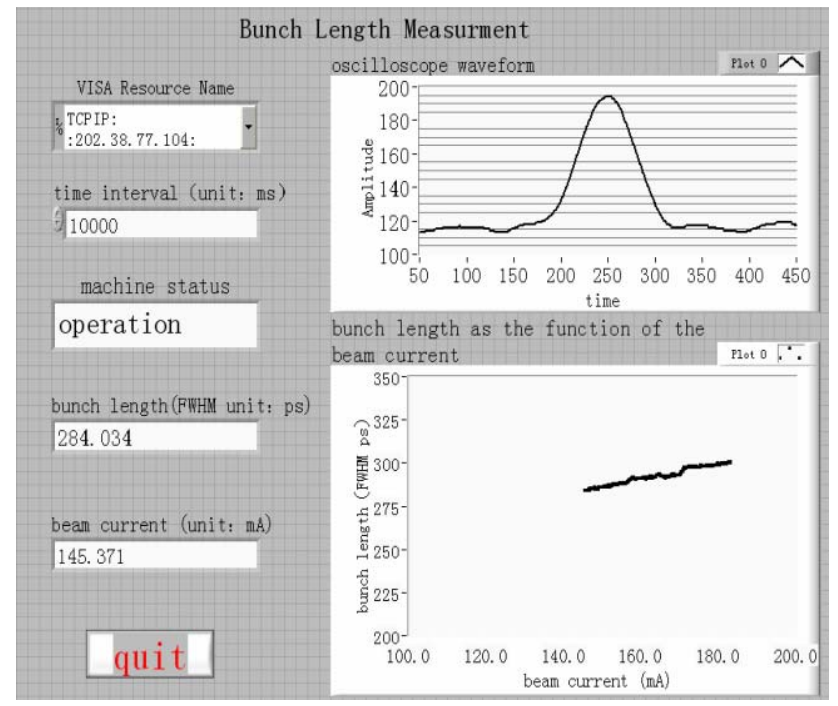

Figure 3: Interface of bunch length measurement.

\section{Results}

When the beam current is $150 \mathrm{~mA}$, the waveform of the electrical signal observed directly from the oscilloscope is displayed in Fig.4.

T03 Beam Diagnostics and Instrumentation 


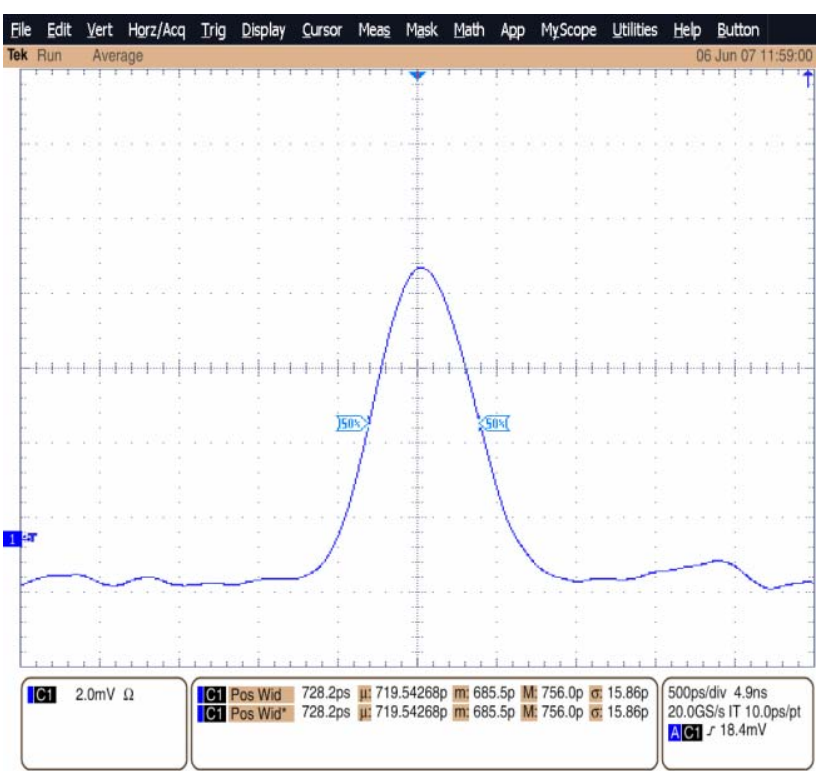

Figure 4: Signal waveform when beam current is $150 \mathrm{~mA}$.

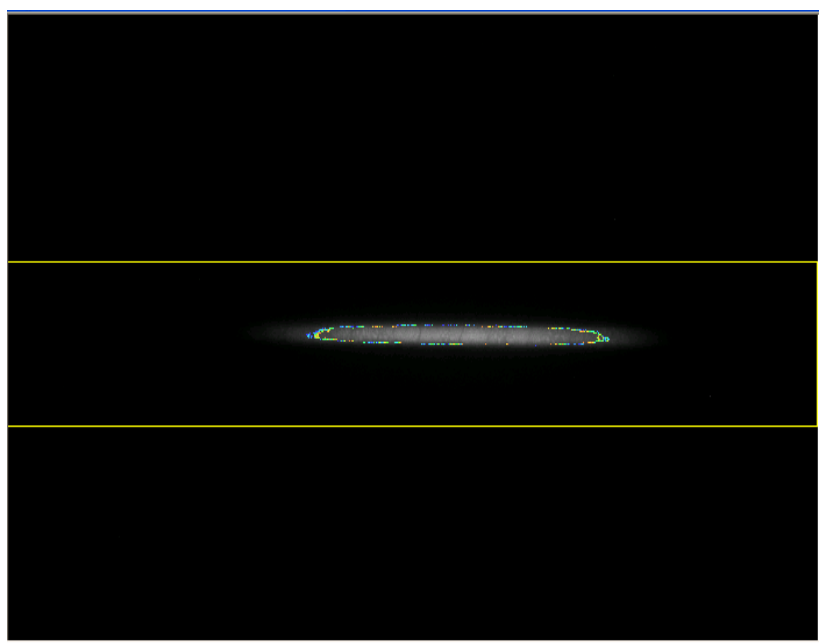

Figure 5: Result from streak camera with horizontal time range $1.96 \mathrm{~ns}$ when beam current was $145 \mathrm{~mA}$.

The result from the streak camera ${ }^{[8]}$ when beam current was $145 \mathrm{~mA}$ is illustrated in Fig.5. In Fig.5, the streak camera worked in the single sweep mode, its horizontal range was $1.96 \mathrm{~ns}$, and the time resolution was $8 \mathrm{ps}$. We got the bunch length from Fig.5 through special software supported by the streak camera.

Under different beam current, the bunch length is measured using the oscilloscope and the streak camera, as shown in Fig.6. In Fig.6, the solid curve represents the calculated bunch length from the oscilloscope and the squares represent values measured by the streak camera. It is seen that the bunch length measured by the oscilloscope agrees well with the value by the streak camera in Fig.6. So, this measuring technique of bunch length in time domain provides means of permanent monitoring of the bunch length without the continuous operation of a streak camera.

The natural bunch length was calculated, the value is 93.6ps with the synchrotron frequency $37.5 \mathrm{KHz}$. We can see the bunch lengthening as the beam current increases clearly from Fig.6.

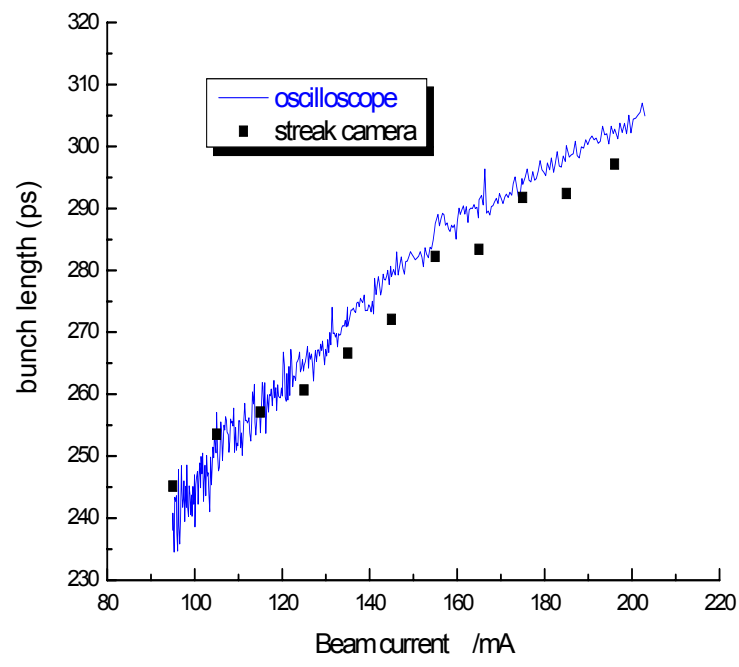

Figure 6: Bunch lengthening with the beam current.

\section{CONCLUSIONS}

We measured the dependence of bunch length on beam current in full fill pattern and compared the results form the oscilloscope and the streak camera. The experimental results proved that the bunch length increases as the beam current increases. Future work will measure the single bunch and the bunch shape transformation.

\section{REFERENCES}

[1] T. Ieiri, "Measurement of Bunch Length Based on Beam Spectrum in the KEKB", Proceedings of EPAC'00, 2000.8, p. 1735.

[2] Z. Zhao, Z.Y. Guo et al., "The Bunch Length Measurement of BEPC with a Streak Camera", Proceedings of APAC'01, 2001.9, p.598.

[3] M. Ferianis, M. Pros, "A new frequency-domain method for bunch length measurement", Proceedings of PAC'97, 1997.5, p. 2011.

[4] B.G. Sun, J.H. Wang et al., "Bunch length measured by $20 \mathrm{GHz}$ digital sampling oscilloscope", Atomic Energy Science and Technology, 1996, p.368.

[5] B.G. Sun, L.K. Chen et al., "Bunch Length Measurement and Its Lengthening in HLS", Proceedings of PAC'99, 1999.4, p. 2184.

[6] FEMTO Co, Datasheet of HSA-XS-2G-IN.

[7] Tektronix Co, TDS7000B datasheet.

[8] J.G. Wang, B.G. Sun et al., "The streak camera system in HLS", Proceedings of 27th HSPP, 2006.10, Vol. 6279, p. 62792G:1-7. 